\title{
X-ray tracking of structural changes during a subnanosecond solid-solid phase transition in cobalt nanoparticles
}

Vester, Peter; Christensen, Morten; Biasin, Elisa; Mariager, Simon O.; Newby, Gemma; Khakhulin, Dmitry; Zontone, Federico; Wulff, Michael; Feidenhans'I, Robert Krarup; Nielsen, Martin M.

Total number of authors:

11

Published in:

Physical Review B

Link to article, DOI:

10.1103/physrevb.100.245425

Publication date:

2019

Document Version

Publisher's PDF, also known as Version of record

Link back to DTU Orbit

Citation (APA):

Vester, P., Christensen, M., Biasin, E., Mariager, S. O., Newby, G., Khakhulin, D., Zontone, F., Wulff, M., Feidenhans'l, R. K., Nielsen, M. M., \& Haldrup, K. (2019). X-ray tracking of structural changes during a subnanosecond solid-solid phase transition in cobalt nanoparticles. Physical Review B, 100(24), [245425]. https://doi.org/10.1103/physrevb.100.245425

\section{General rights}

Copyright and moral rights for the publications made accessible in the public portal are retained by the authors and/or other copyright owners and it is a condition of accessing publications that users recognise and abide by the legal requirements associated with these rights.

- Users may download and print one copy of any publication from the public portal for the purpose of private study or research.

- You may not further distribute the material or use it for any profit-making activity or commercial gain

- You may freely distribute the URL identifying the publication in the public portal 


\title{
X-ray tracking of structural changes during a subnanosecond solid-solid phase transition in cobalt nanoparticles
}

\author{
Peter Vester $\odot,{ }^{1}$ Morten Christensen $\odot,{ }^{1}$ Elisa Biasin,,${ }^{1,2}$ Simon O. Mariager, ${ }^{3}$ Gemma Newby $\odot,{ }^{4}$ Dmitry Khakhulin, ${ }^{4,5}$ \\ Federico Zontone, ${ }^{4}$ Michael Wulff, ${ }^{4}$ Robert Feidenhans' $1,{ }^{3,5}$ Martin M. Nielsen $\odot,{ }^{1}$ and Kristoffer Haldrup ${ }^{1}, * *$ \\ ${ }^{1}$ Technical University of Denmark, Department of Physics, Fysikvej 307, DK-2800 Kongens Lyngby, Denmark \\ ${ }^{2}$ PULSE Institute, SLAC National Accelerator Laboratory, Stanford University, Menlo Park, California 94025, USA \\ ${ }^{3}$ University of Copenhagen, Niels Bohr Institute, Blegdamsvej 17, 2100 Copenhagen \\ ${ }^{4}$ ESRF-The European Synchrotron, 38043 Grenoble, France \\ ${ }^{5}$ European XFEL, D-22869 Schenefeld, Germany
}

(Received 22 August 2019; published 23 December 2019)

\begin{abstract}
This work presents measurements of laser-induced solid-solid phase transitions in Co nanoparticles, using time-resolved wide-angle $\mathrm{x}$-ray scattering with $<100$-ps time resolution. A comparison of the experimental results with scattering simulations indicates a phase transition from the as-synthesized $\epsilon$ phase into a mixed phase of $2 / 3 \mathrm{hcp}$ and $1 / 3 f c c$ character during the first $100 \mathrm{ps}$. The measured scattering signal observed at the shortest investigated time delay of $20 \mathrm{ps}$ has less scattering intensity at the dominant $f c c / h c p$ Bragg peak positions compared to the difference signal acquired at $100 \mathrm{ps}$. This suggests the formation of a partially disordered solid intermediate phase on the sub-100-ps timescale.
\end{abstract}

DOI: 10.1103/PhysRevB.100.245425

\section{INTRODUCTION}

Colloidal metal nanoparticles have been the subject of intense interest in areas such as materials science, biotechnology, chemical synthesis, diagnostic imaging, and catalysis as well as for information storage [1-5]. The research demonstrates significant potential for applications due to the particles unique properties which can, to a large extent, be tailored via different synthesis routes [6-10]. The structure and shape of the nanoparticles is essential for their chemical and magnetic behavior and much effort has been put into techniques to control these properties, both during synthesis and after [11]. As an examples of postsynthesis modification of nanoparticle properties utilizing laser illumination of nanoparticle solutions, illumination with intense nanosecond laser pulses exciting plasmon modes at $532 \mathrm{~nm}$ has been shown to give rise to highly monodisperse nanoparticles in the case of $\mathrm{Au}$ [12]. For characterizing these processes in more detail, the structural dynamics during pulsed laser heating, melting and resolidification of $\mathrm{Au}$ nanoparticles have been measured with time-resolved $\mathrm{x}$-ray scattering experiments [13-15], but the direct structural dynamics of a solid-solid phase transition has to our knowledge not been reported. Here we report on synchtrotron-based structural studies of a solid-solid phase transformation on the sub-100-ps timescale in Co nanoparticles.

The crystal structure of bulk Co is hexagonal close packed (hcp) at temperatures below $720 \mathrm{~K}$ and face-centered cubic (fcc) up to the melting point at $1770 \mathrm{~K}$ [16] with a martensitic solid-solid $h c p-f c c$ phase transition, i.e., stress-induced and

*Corresponding author: hald@fysik.dtu.dk accomodated by Shockley partial dislocation motions $[17,18]$. However, the as-synthesized particles in this study exhibit a metastable $\epsilon$ phase, a complex cubic primitive resembling the $\beta$-Mn cell structure, and for Co nanoparticles only observed in samples produced via the solution-phase synthesis route [19-21] as described in detail by Zacharaki et al. [22]. In general, the small size of the particles and their large surfaceto-volume ratio often leads to different phase diagrams than for the corresponding bulk materials. In the present case, the $\epsilon$ phase in Co nanoparticles has previously been shown by annealing to irreversibly transform to the $h c p$ phase at $\approx 600 \mathrm{~K}$ and $f c c$ at $\approx 800 \mathrm{~K}[20,23-25]$.

The crystal structure of Co nanoparticles at room temperature has been demonstrated to depend on the synthesis route and processing history of the sample due to almost isoenergetic crystal phases [26]. Apart from the metastable $\epsilon$ phase produced by the above-mentioned solution-phase synthesis route, for small nanoparticles $(<20 \mathrm{~nm})$ the $f c c$ structure appears to be favored at room temperature in nanoparticles created by sputtering, with mixed-phases observed for mediumsized particles (30-40 nm) and $h c p$ structure for large-size particles $(>40 \mathrm{~nm})$ [27]. Studies of small Co nanoparticles $(2-11 \mathrm{~nm})$ produced by high-temperature crystallization have suggested mixed phases dominated by the $h c p$ structure at room temperature, with a transition to $f c c$ at $800 \mathrm{~K}$ [23].

A study of larger Co nanoparticles $(\sim 100 \mathrm{~nm})$ using in situ heating in a transmission electron microscope setup showed a mixed $f c c / h c p$ phase at room temperature, an observation which was concluded to arise from incomplete transformation to the $h c p$ structure during rapid cooling of the nanoparticles formed via a pulsed wire evaporation synthesis method [28]. In the limit of slow cooling, purely $f c c$ nanoparticles can be formed via ion implantation and annealing in solid matrices, 
following which irradiation by energetic heavy ions can induce a transformation to either an amorphous phase [29] or to a crystalline $h c p$ phase, a transformation surmised to take place without the involvement of a liquid-phase intermediate but rather arising from a large transient shear stress due to local heating [26,30]. Further, as also discussed in Ref. [26], on heating the phase transformation from $h c p$ to $f c c$ is generally complete, whereas the $f c c$-to-hcp transformation on cooling is generally only partial, in line with very recent theoretical studies of the effect of cooling rates on the microstructural evolution of nanocrystalline Co [31]. In the context of these studies the pressure, heating/cooling rate, grain size, and impurities have been identified as sources of the differing results regarding phase transformations [26,28,31].

\section{EXPERIMENTAL SETUP}

In the present experiments, the Co nanoparticles were synthetized using the organometallic method [19-21] to produce a monodisperse distribution of spherical particles with a diameter of about $7 \pm 2 \mathrm{~nm}$ (full width at half maximum, FWHM) as measured by transmission electron microscopy (TEM) (Fig. 1).

Following initial synthesis, the reaction mixture was washed with ethanol before the nanoparticles were redissolved in 2-propanol. The initial concentration of Co in the
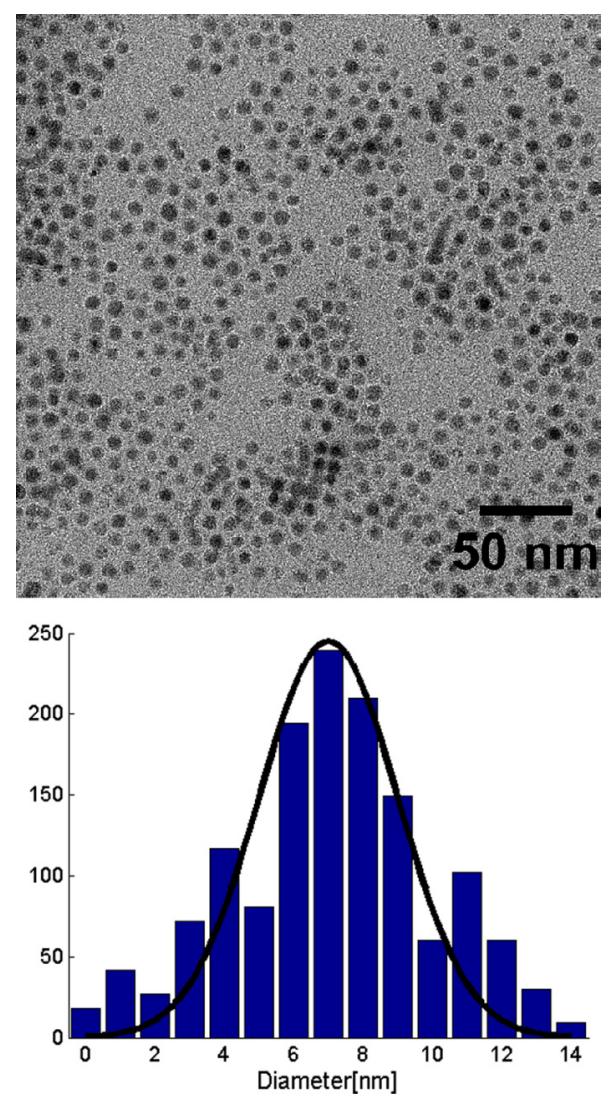

FIG. 1. An example of a TEM image of the spherical Co nanoparticles along with the measured size distribution from several TEM images of $\approx 1500$ particles. The black curve represents a Gaussian distribution with a center at $7 \mathrm{~nm}$ and a FWHM of $4 \mathrm{~nm}$. reactants was $83 \mathrm{mM}$, and for the x-ray experiments $70 \mathrm{ml}$ of this sample was diluted by a further $35 \mathrm{ml}$ of 2-propanol. Just before the $\mathrm{x}$-ray experiments, additional surfactants were added to the solution ( $\sim 40 \mathrm{mg}$ of trioctylphosphine oxide and $\sim 100 \mathrm{mg}$ of oleic acid) to improve the nanoparticle stability under the experimental conditions. As discussed in further detail below, the structural analysis indicates a final nanoparticle concentration of $0.35 \mu \mathrm{M}$, corresponding to $5 \mathrm{mM}$ Co atoms.

To investigate the dynamics of the solvated nanoparticles, we utilized a well-established time-resolved pump-probe wide-angle $x$-ray scattering (TR-WAXS) approach at the ID09 beamline of the European Synchrotron Radiation Facility (ESRF) as schematically illustrated in Fig. 2.

The experimental procedure for such solution-state investigations has been described in detail previously $[32,33]$. Briefly, the sample is excited by a short ( $<2$ ps) 390-nm laser pulse (pump) followed by a $\sim 100$-ps $18.2-\mathrm{keV}$ pink-beam (FWHM $\sim 3 \%$ ) $x$-ray pulse (probe) at a well-defined time delay $\Delta t$ after the photoexcitation event. A high-speed chopper is used to isolate one $\mathrm{x}$-ray pulse from the synchrotron for each recorded pump-probe event $(\sim 1-\mathrm{kHz}$ repetition rate, determined by the optical laser system). The recorded x-ray scattering from this pump-probe event gives a snapshot of the structural configuration after laser excitation at a single time delay. By combining snapshots at different time delays, the dynamics can be followed in time.

The solution prepared as described above was flowing through a vertical free flowing flat liquid jet of $300-\mu \mathrm{m}$ thickness with a flow rate sufficient to fully replace the sample between successive pump-probe events. The nearly collinear laser beam with $40-\mu \mathrm{J}$ pulses and a circular spot size of $\sim 200-\mu \mathrm{m}$ diameter $\left(64-\mathrm{mJ} / \mathrm{cm}^{2} /\right.$ pulse $)$ and the $\mathrm{x}$-ray beam with an estimated spot size of $100 \times 60 \mu \mathrm{m}$ were spatially and temporally overlapped in the center of the liquid jet.

The TR-WAXS signal was recorded in the forward direction by a large-area FReLoN (Fast-Readout, Low-Noise) detector [34] positioned $4.6 \mathrm{~cm}$ behind the sample. Integration times were $4 \mathrm{~s}$ and several hundred such exposures were acquired per time point. The scattering images were azimuthally integrated to yield one-dimensional $S(Q)$ curves, where $Q=$ $\frac{4 \pi}{\lambda} \sin (\theta)$ is dependent on the scattering angle $\theta$ and the $x$-ray wavelength $\lambda$.

Scattering patterns acquired at $\Delta t=-3 \mathrm{~ns}$ were used as laser-off reference measurements to construct difference signals $\Delta S(Q, t)=S_{\text {on }}(Q, t)-S_{\text {off }}(Q, t)$ as shown in Fig. 3 (left). As such, the difference signals record only what was changed by the laser pulse and the approach therefore additionally serves as an efficient background-suppression method. For the experiments presented here, this is highly beneficial as the signal arising from the Co nanoparticles in the solution is less than $1 \%$ of the total scattering signal (Supplemental Material [35], Fig. S3).

The overall time resolution of the experiment is determined by the width of the x-ray probe pulses, which are $\sim 100 \mathrm{ps}$ long. However, a fast diamond detector can be used to monitor the pump-probe delay with an accuracy of approximately $5 \mathrm{ps}$, enabling "slicing" experiments $[36,37]$ as discussed in further detail below. 


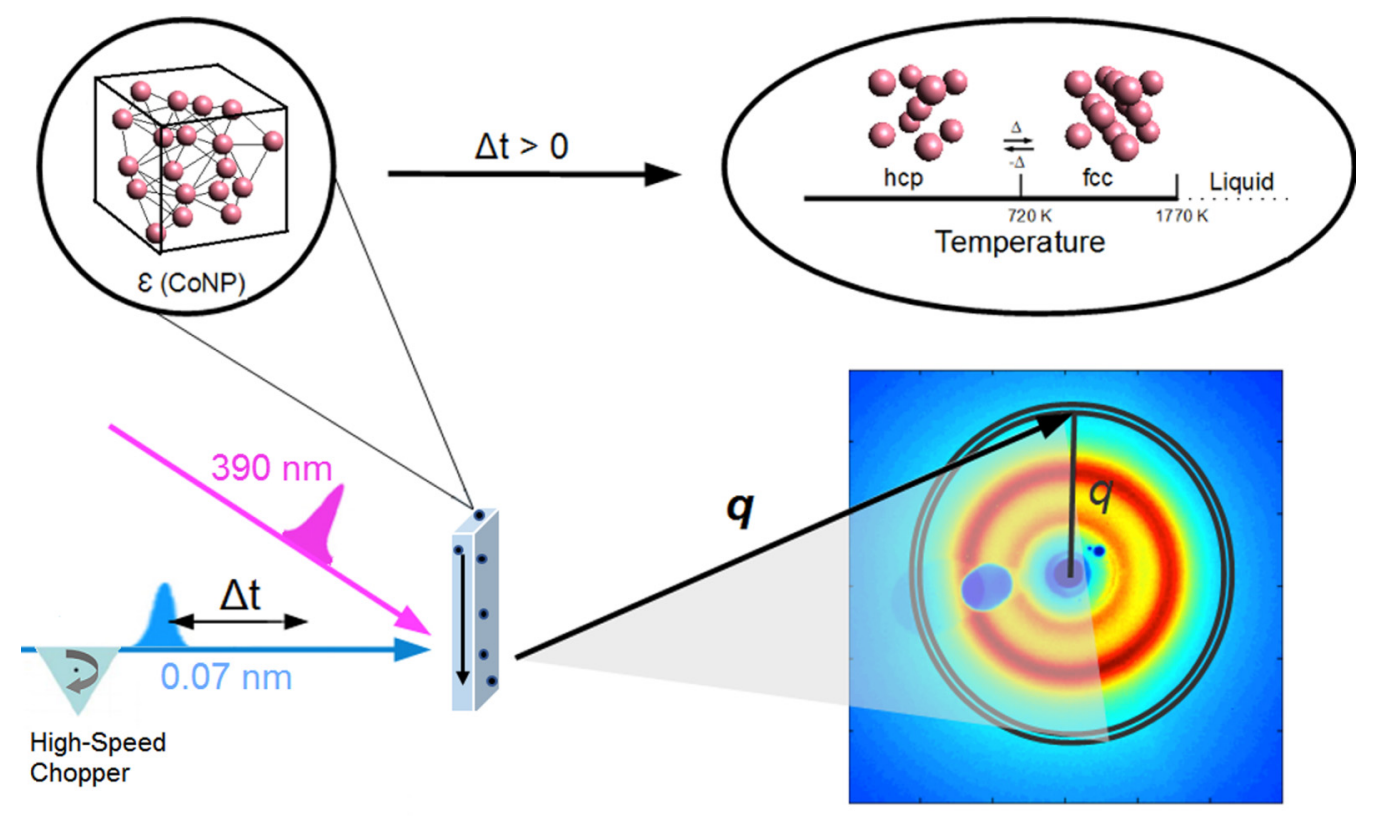

FIG. 2. Schematic illustration of the liquid-sheet TR-WAXS setup at beamline ID09 (ESRF). The experiment is designed such that a fresh sample (Co nanoparticles in 2-propanol) is measured for each pump-probe event. Well-defined time delays between the pump (laser) and probe (x-ray) give subnanosecond time resolution. The as-synthesized particles start out in the $\epsilon$ phase which transforms irreversibly on laser excitation to hexagonal close-packed $(h c p)$ and face-centered cubic $(f c c)$ structure. From bulk values the $h c p-f c c$ phase transition is expected at around $720 \mathrm{~K}$ in a reversible process.

\section{RESULTS}

Figure 3 shows the time-resolved $x$-ray difference scattering signal at different time delays $\Delta t$ after laser excitation as azimuthally integrated $\Delta S(Q, \Delta t)$ curves. These difference signals arise from primarily two different sets of structural changes, namely the difference signal arising from changes
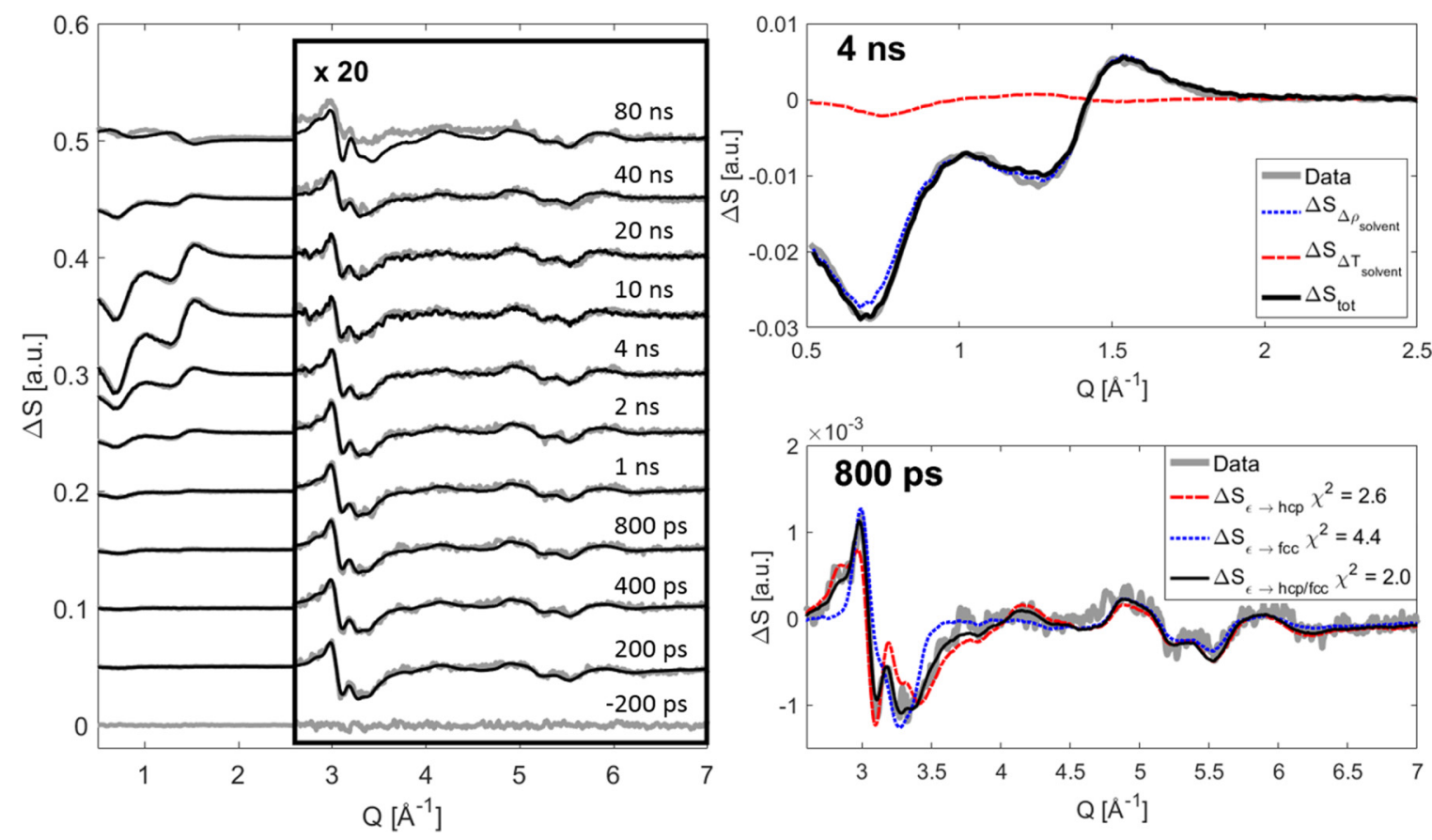

FIG. 3. Left: Measured $\Delta S(Q, \Delta t)$ (gray) compared with the calculated scattering (black) from a model based on a mixed $\epsilon \rightarrow h c p / f c c$ phase transition $\left(\Delta S_{\epsilon \rightarrow h c p} / \Delta S_{\epsilon \rightarrow f c c}\right)$ and changes in the solvent temperature/density $\left(\Delta S_{\rho} / \Delta S_{T_{\text {solv }}}\right)$. Right: Low- $Q$ (top) and high- $Q$ (bottom) spectra shows the contributions at different time delays. The bottom figure also reports the $\chi^{2}$ values from modeling with single and mixed phase transitions. 
in the nanoparticle structure $\left(\Delta S_{\mathrm{NP}}\right)$ and the difference signal arising from changes in the bulk solvent structure $\left(\Delta S_{\text {solv }}\right)$ :

$$
\Delta S_{\text {total }}=\Delta S_{\mathrm{NP}}+\Delta S_{\text {solv }} .
$$

Qualitatively, $\Delta S_{\text {solv }}$ arises from structural changes in the bulk solvent which are due to changes in temperature and density of the bulk solvent following photoexcitation of the sample [38]. This contribution to the difference signal is observed to be strongest around and below the so-called liquid peak at $Q=1.8 \AA^{-1}$ (Supplemental Material [35], Fig. S3) with almost no solvent difference signal above $Q=2.5 \AA^{-1}$ in full agreement with previous investigations of 2-propanol [39]. The signal arising from the Co nanoparticle dynamics dominates the difference signal at $Q$ values exceeding $3 \AA^{-1}$, allowing robust separation of the solvent- and nanoparticle contributions in the analysis. At timescales longer than $100 \mathrm{~ns}$ (not shown) the signal becomes dominated by a strong signal surmised to arise from surface evaporation of the sample jet. At low $Q$, the time evolution is observed to be dominated by a feature growing in on a nanosecond timescale and disappearing on a 10-ns timescale. At higher $Q$, strong features at $Q=3 \AA^{-1}$ and weaker ones at $Q=5.5 \AA^{-1}$ appear promptly at the 200-ps time step and remain essentially constant in the entire time range shown. The $Q=3 \AA^{-1}$ features in the difference scattering signals can be directly compared with the (221), (310), and (311) peaks shown in the simulated $\epsilon$-phase nanoparticle diffraction signals in Supplemental Material Fig. S1 [35] (see also Ref. [40] therein), and the $Q=5.5 \AA^{-1}$ features appear related to the (510) and (520) peaks. As such, these qualitative observations are consistent with the initial photoexcitation of the Co nanoparticles leading to a change in structure on a $<200$-ps timescale followed by energy transfer to the surrounding solvent medium in agreement with previous nanoparticle studies using the same methodology [14]. Below we present a more detailed analysis of these general observations, leading to the model fits also shown in Fig. 3.

On the 100-ps to $-\mu$ s timescales the solvent contribution has been shown to be well described by a linear combination of two difference signals which arise from the molecular rearrangement due to solvent temperature increase $\left(\left.\Delta T_{\text {solv }} \frac{\partial S}{\partial T}\right|_{\rho}\right)$ and density changes $\left(\left.\Delta \rho \frac{\partial S}{\partial \rho}\right|_{T}\right)$, respectively [38]. These terms were measured in a separate experiment on dye-heated 2-propanol following the procedures outlined in the work of K. Kjær et al. [39].

The difference scattering signal arising from photoexcited nanoparticle dynamics may come from several connected structural changes. Common for all of these possible contributions to the difference signal is that the ground-state scattering pattern $\left(S_{\text {off }}\right)$ is a powder scattering pattern from randomly oriented 7-nm Co nanoparticles in the $\epsilon$ phase. This was simulated from a Gaussian distribution (FWHM = $4 \mathrm{~nm}$ ) of $d=7 \mathrm{~nm}$ Co particles using the full pink-beam U17 undulator spectrum peaked at $18.2 \mathrm{keV}$ using the Debye equation, $S(Q)=\sum_{i j} f_{i} f_{j} \frac{\sin Q r_{i j}}{r_{i j}}$, where $f$ denotes the atomic form factor and $r_{i j}$ the distance between atoms $i$ and $j$.

Following photoexcitation and energy transfer from the electron system to the crystal lattice $(<10 \mathrm{ps})$ several structural transformations are simultaneously active: Lattice disorder and lattice expansion due to heating, as well as possible phase transformations from the initial $\epsilon$ phase to either a $f c c$ phase, a $h c p$ phase, a disordered/liquid phase or a mixture of these. A transformation from the $\epsilon$ phase to a disordered phase would give rise to only negative features in the difference signal (diffraction peaks disappearing following photoexcitation) as discussed in further detail below, and as evident from Fig. 3, positive features are present at all the time delays shown and we therefore focus the initial analysis on the possible solid-solid phase transformations.

To simulate the difference signal arising from solid-solid structural transformations, the scattering patterns for both $f c c$ and hcp 7-nm nanoparticles were calculated (Fig. S2 in the Supplemental Material [35]). As for the $\epsilon$ phase, this was done via the Debye equation, but for each of the two possible structural transformations $(\epsilon \rightarrow h c p$ and $\epsilon \rightarrow f c c)$ a two-dimensional set of difference signals were calculated corresponding to a range of values for the nanoparticle lattice expansion $(\Delta a)$ and for temperature increase $\left(\Delta T_{\mathrm{NP}}\right)$, with the latter calculated via the Debye-Waller factor (Fig. S5 in the Supplemental Material [35] and Ref. [41] therein).

From these considerations and suppressing the $Q$ dependence for clarity of presentation, each of the two terms in Eq. (1) can be calculated through the following two expressions:

$$
\begin{gathered}
\Delta S_{\text {solv }}=\left.\Delta T_{\text {solv }} \frac{\partial S}{\partial T_{\text {solv }}}\right|_{\rho}+\left.\Delta \rho \frac{\partial S}{\partial \rho}\right|_{T_{\text {solv }},} \\
\Delta S_{\mathrm{NP}}=\alpha S_{h c p}\left(\Delta T_{\mathrm{NP}}, \Delta a\right)+\beta S_{f c c}\left(\Delta T_{\mathrm{NP}}, \Delta a\right)-(\alpha+\beta) S_{\epsilon},
\end{gathered}
$$

rendering a full model with six parameters $\left(\alpha, \beta, \Delta T_{\mathrm{NP}}, \Delta a, \Delta T_{\mathrm{solv}}, \Delta \rho\right)$, where $\alpha$ and $\beta$ denote the relative fractions undergoing a structural change. As the solvent contribution dominates at low- $Q$ and the Co nanoparticle term at high- $Q$ (see Fig. 3), the solute and solvent model parameters were found to be only weakly correlated. Least-squares fitting was used to fit Eqs. (1)-(3) to the acquired difference scattering signals. Figure 3 (left) shows the difference scattering signals acquired (gray curves) and the simulated difference signals (black curves) for 10 time delays in the range from 200 ps to $80 \mathrm{~ns}$. Figure 3 (right) shows the model fit to the data at two time delays in more detail. At $800 \mathrm{ps}$, the difference signal at $Q>2.5 \AA^{-1}$ is dominated by the difference signal arising from structural changes in the nanoparticles, in particular the disappearance of the (221), (310), and (311) peaks of the $\epsilon$ structure around $Q=3$ $-3.5 \AA^{-1}$ which is accompanied by the grow-in of the $f c c$ and $h c p$ diffraction peaks slightly below $Q=3 \AA^{-1}$. From close inspection of the difference signal in the $Q=2.8-3.5 \AA^{-1}$ region it is evident how a model comprising a transformation to mixed-phase nanoparticles best captures all features of the signal - a complete transformation to $h c p$ will give rise to a difference signal with a shoulder at $Q=2.8 \AA^{-1}$ and a very sharp negative feature at $Q=3.1 \AA^{-1}$, features absent from the $\epsilon \rightarrow f c c$ difference signal. However, inclusion of the $\epsilon \rightarrow$ $f c c$ difference signal allows the model to much better capture the width of the negative $Q=3.1 \AA^{-1}$ feature. Figure 3 (right bottom panel) illustrates this at 800 ps and shows a lower $\chi^{2}$ value (2.0) for a mixed phase transition compared to complete $h c p\left(\chi^{2}=2.6\right)$ or $f c c\left(\chi^{2}=4.4\right)$ transitions. 

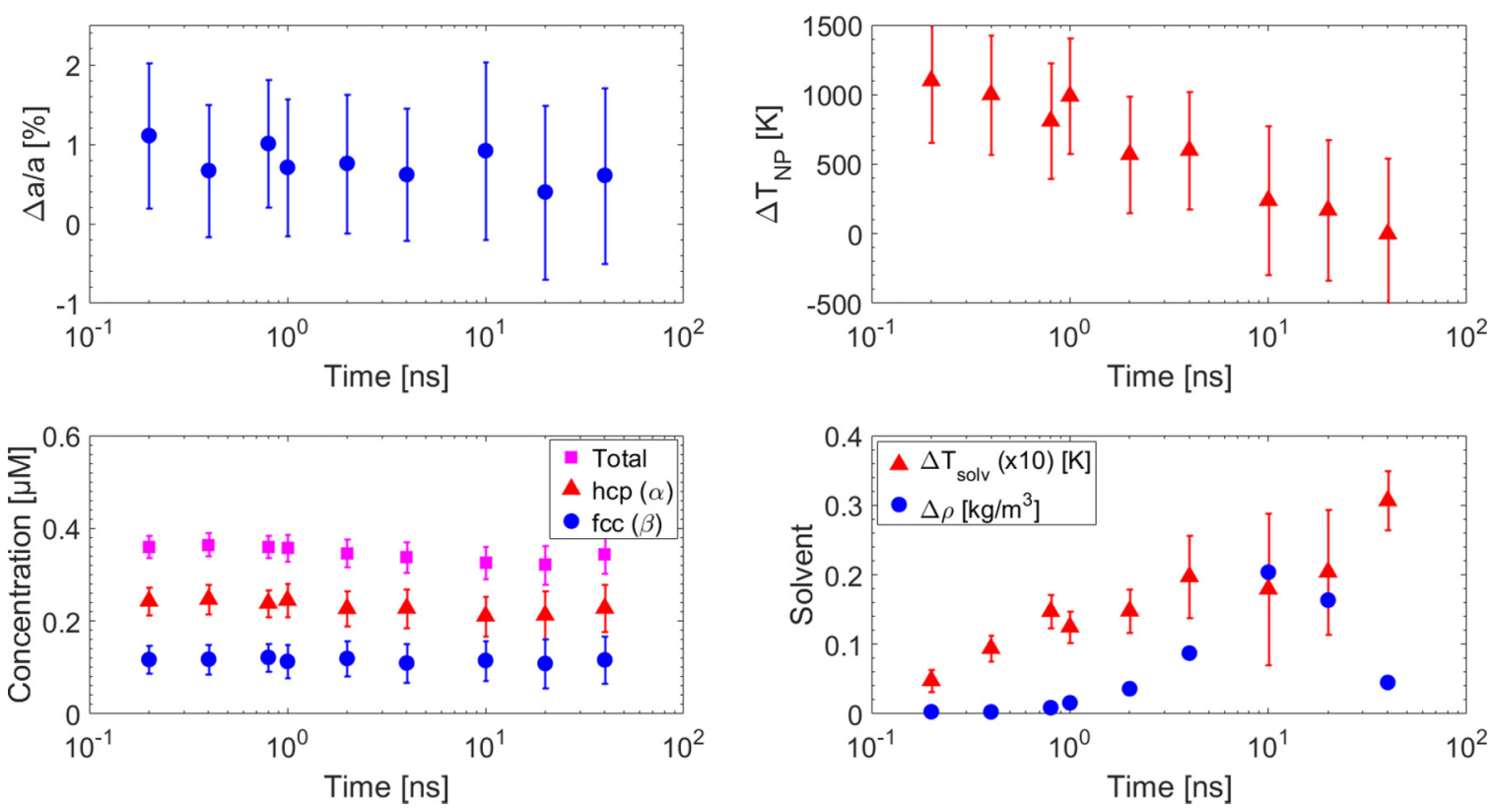

FIG. 4. Best-fit model parameters using the model in Eqs. (1)-(3) to fit each time delay. The concentration of $h c p / f c c$ ( $\alpha$ and $\beta$ parameters) Co nanoparticles is constant at these timescales and the cooling is observed through a decrease in $\Delta T_{\mathrm{NP}}$. The cooling of the particles is accompanied by an increase of the solvent temperature $\left(\Delta T_{\text {solv }}\right)$. An increase in solvent density $(\Delta \rho)$ is observed at intermediate time steps, which is associated to hot vapor bubble formation around the particles on a nanosecond timescale. The values at -200 ps (before excitation) are not included in the plots but they converge to zero when no difference signal is observed (Fig. 3, left).

At a time delay of $4 \mathrm{~ns}$, the difference signal shape in the high- $Q$ region of the data is essentially the same as at $800 \mathrm{ps}$ (Fig. 3, left) but a strong negative feature has evolved at low $Q$. Figure 3 (right top panel) shows this in more detail, and in general all features of the difference signal are well captured by the solvent response in the model, $\Delta S_{\text {solv }}$, and arises from an increase in solvent temperature as well as increase in solvent density. As discussed in further detail below, this observation is assigned to the formation of vapor bubbles around the nanoparticles as previously seen in similar studies of photoexcited nanoparticles in solution [42,43].

Figure 4 shows the time evolution of all the six free parameters in the model applied to fit the data. In general we find that the difference signal at all time delays is well captured (Fig. 3) by a $\sim 2 / 3 \epsilon \rightarrow h c p(\alpha)$ and $\sim 1 / 3 \epsilon \rightarrow$ $f c c(\beta)$ transformation (Fig. 4, lower left panel) with the lattice constant of the final structural phases being on the order of $1 \%$ larger than determined for bulk samples of Co. The temperature increase of the nanoparticles is determined to be initially on the order of $1000 \mathrm{~K}$ (for reference, the melting temperature of bulk $\mathrm{Co}$ is $1770 \mathrm{~K}$ ), decreasing to ambient temperature on a 1 - to 10 -ns timescale (Fig. 4, upper right panel). This temperature decrease of the nanoparticles is followed by a 0.3 degrees temperature increase of the bulk solvent, along with a transient increase of $0.2 \mathrm{~kg} / \mathrm{m}^{3}(0.2 \%$ ) in the average density of the solvent in the probed volume.

Turning next to the shortest timescales investigated in this set of experiments, Fig. 5 shows difference scattering signals recorded at $\Delta t=20 \mathrm{ps}$ and $100 \mathrm{ps}$, using the "slicing mode" where the short excitation pulse arrives at the sample position within the envelope of the x-ray pulse. The insert illustrates how at $100 \mathrm{ps}$ the full $\mathrm{x}$-ray pulse probes the photoexcited sample (shaded areas), whereas at nominal $\Delta t=0$ only the latest $50 \mathrm{ps}$ of the $\mathrm{x}$-ray pulse probes photoexcited sample, leading to a smaller difference signal as discussed in full detail in Refs. [36,37,44]. The asymmetric pulse shape arises from wake-field effects in the synchrotron beam pipe [45]. To compensate for the down-scaling of the difference signal magnitude due to only part of the probe pulse interacting with photoexcited sample, the difference signals shown in Fig. 5 have been scaled accordingly and as such can be directly compared. $\Delta S(Q, \Delta t=100 \mathrm{ps})$ is almost identical to $\Delta S(Q, \Delta t=200 \mathrm{ps})$ shown in Fig. 3 (left), whereas $\Delta S(Q, \Delta t=20 \mathrm{ps})$ has a significantly reduced magnitude

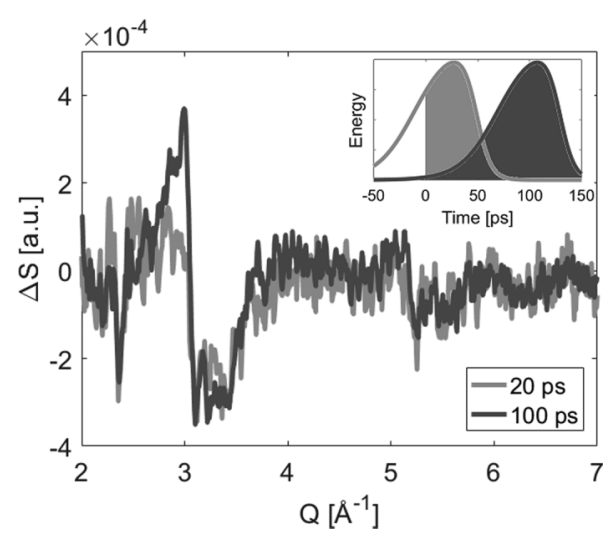

FIG. 5. Scattering difference signals at 20 and 100 ps acquired with improved time resolution but lower signal intensity by using fast diamond detector and a "slicing" scheme. A significantly different signal shape is observed at around $Q=2.9-3.0 \AA^{-1}$ at $20 \mathrm{ps}$ compared to the 100-ps difference signal. The insert illustrates the part of the temporal $\mathrm{x}$-ray profile that records the laser excited state for (nominally) $0 \mathrm{ps}$ and $100 \mathrm{ps}$. 
of the positive features around $Q=2.9 \AA^{-1}$, indicating a possible disordered, short-lived precursor state to the $f c c / h c p$ final state(s).

\section{DISCUSSION}

Comparison of the calculated difference scattering with the measurements at $0.2-40 \mathrm{~ns}$ (Fig. 3) indicates a mixed $\epsilon \rightarrow h c p / f c c(2 / 3$ to $1 / 3)$ phase transition following laser excitation. No significant change of the $h c p / f c c$ ratio was observed during the time after the initial excitation indicating that the initially created $h c p / f c c$ structure is stable also following cooling to room temperature.

As reviewed in the Introduction, most previous studies of phase transitions in $\epsilon$ Co nanoparticles were carried out by annealing and resulted in a complete phase transition to $h c p$ at around $570 \mathrm{~K}$ and to $f c c$ at around $770 \mathrm{~K}$. The creation of a mixed phase of $1 / 3 f c c$ and $2 / 3 \mathrm{hcp}$ structures is not in contradiction to these results as the near-instantaneous heating by an ultrafast laser pulse is fundamentally different from annealing over a longer time. Recent computational results, however, point to the quenching rate rather than the heating rate being key to determine the properties of the new $f c c / h c p$ phases appearing after ultrafast heating and cooling [31]. In their study, quench rates from $10^{10}$ to $10^{12} \mathrm{~K} / \mathrm{s}$ were investigated, directly comparable to the quench rate of $10^{11} \mathrm{~K} / \mathrm{s}$ in the present study, and in this regime mixed-phase $f c c / h c p$ nanostructures were observed to form, in agreement with the results presented above. Further, the timescale for forming $f c c / h c p$ clusters with $\sim 10^{4}$ atoms (roughly corresponding to the 7-nm nanoparticles in this study) was estimated from these MD simulations to be $\sim 50$ ps in good agreement with the results presented in Fig. 5.

The appearance of the new crystal phases observed in the difference scattering signals in Figs. 3 to 5 take place on a significantly faster timescale than what has been reported for recrystallization following photoexcitation to a liquid phase. Plech et al. [13] reported a timescale of 0.5-5 ns for resolidification of Au nanoparticles at laser fluences below the ablation threshold, which is in good agreement with a recent study of small $(R=1.5-4 \mathrm{~nm})$ CdSe nanoparticles at similar fluences [46], where timescales of 100 to 300 ps were estimated for $R=1.5 \mathrm{~nm}$ and $R=3.8 \mathrm{~nm}$ nanoparticles, respectively.

The observed particle cooling time of $\sim 5 \mathrm{~ns}$ as estimated from the Debye-Waller factors (as opposed to most previous studies, which estimated the temperature from the lattice expansion $\Delta a / a$ ) is somewhat faster than the $10 \mathrm{~ns}$ Plech et al. [13] determined for 36-nm gold particles, as expected from the smaller particle size and slower than the $1 \mathrm{~ns}$ determined by Kirschner et al. for 3-nm CdSe nanoparticles [46]. As such, the present estimate for the nanoparticle cooling rate is in good agreement with these studies, as the nanoparticle size of $7 \mathrm{~nm}$ in our study is intermediate to the nanoparticle sizes in those two previous studies. In all cases, the cooling time is longer than expected from continuum theory [47] and from nanoparticles in solid matrices [48], an observation ascribed to the formation of insulating vapor bubbles around the photoexcited nanoparticles as energy is transferred to the surrounding solvent medium [43]. This nanobubble formation is readily observable and can be studied in the small angle $\mathrm{x}$-ray scattering (SAXS) region [14], and in the present wideangle $\mathrm{x}$-ray scattering study the effect of the bubble formation can be seen as a transient increase in the solvent density as observed in Figs. 3 and 4. The timescale for the bubble collapse observed in this experiment is somewhat slower than what has been observed for $9 \mathrm{~nm}(<1 \mathrm{~ns})$ and $36 \mathrm{~nm}(\approx 2 \mathrm{~ns})$ $\mathrm{Au}$ particles in water. This point toward factors such as a different solvation response in 2-propanol compared to water, potentially due to, e.g., lower heat capacity resulting in bigger bubbles, but elucidating these effects will require a dedicated set of experiments.

\section{CONCLUSIONS AND OUTLOOK}

Time-resolved x-ray wide-angle scattering was successfully performed on 7-nm Co nanoparticles in solution with a time resolution of $100 \mathrm{ps}$ to measure the structural dynamics following photoexcitation of nanoparticles synthesized in the $\epsilon$ phase. Through a comparison with simulated data incorporating effects of both structural phase transitions, lattice expansion and heating as well as solvent dynamics, our structural analysis indicates a phase transition from the $\epsilon$ phase into a mixed phase of $2 / 3 \mathrm{hcp}$ and $1 / 3 \mathrm{fcc}$ structure. Preliminary data on the $\sim 20$-ps timescale indicates that a disordered intermediate is formed but with the prompt $(<100 \mathrm{ps})$ appearance of the mixed final phase, suggesting that this intermediate should be considered as disordered rather than liquid. The solvent scattering signal showed creation of hot solvent gas bubbles around the particles on the nanosecond timescale. This effectively reduced the cooling time by at least one order of magnitude, in agreement with earlier results on $\mathrm{Au}$ nanoparticles [42,43] and suggests a possibility for controlling the cooling rates (and thus final microstructure) by choosing solvents with higher boiling points and/or higher specific heat of evaporation such as, e.g., water.

With the ongoing improvements in synchrotron performance and instrumentation, several avenues are opening up for further studying phase transformations in nanoparticle systems. In particular, increased brilliance and an extended energy range following machine upgrades [49] will allow studies with a larger $Q$ range and utilizing quasimonochromatic rather than pink-beam $x$-rays, greatly increasing the fidelity of the analysis and potentially enabling further analysis of the observed peak broadening here ascribed solely to an increase in nanoparticle temperature. Such high-resolution studies suggest the possibility of discerning the formation of various microstructural phases [31] as a function of incident fluence and quench rates. On the $<50$-ps timescale, x-ray free electron lasers now present an opportunity to study the structural dynamics on the very earliest timescales, from lattice heating via electron-phonon couplings through the formation of potential disordered precursor states to the final, fully crystallized, product state(s). Analysis tools such as $\mathrm{x}$-ray cross correlation analysis (XCCA) allow for measuring ultrafast structural anisotropy induced by the polarized laser pulses [50]. As such, the methodology developed for the present study outlines a path toward studying not only the specific Co nanoparticle system studied here but also diffusion-less (martensitic) solid-solid phase transformations and order-disorder/order-order transitions in a wide range of nanoparticle systems. 


\section{ACKNOWLEDGMENTS}

The DTU affiliated authors gratefully acknowledge DANSCATT for funding the beamtime travel expenses. A. O. Dohn and T. Valentin are thanked for bringing the samples to the ESRF facility despite highly adversarial weather conditions in Northern Europe at the time of the experiments. K.H. and M.C. were supported by the Danish National Research Foundations Centre for Molecular Movies.
[1] M. C. Daniel and D. Astruc, Gold nanoparticles: Assembly, supramolecularchemistry, quantum-size-related properties, and applications toward biology, catalysis, and nanotechnology, Chem. Rev. 104, 293 (2004).

[2] S. Ibrahimkutty, J. Kim, M. Cammarata, F. Ewald, J. Choi, H. Ihee, and A. Plech, Ultrafast structural dynamics of the photocleavage of protein hybrid nanoparticles, ACS Nano $\mathbf{5}$, 3788 (2011).

[3] N. A. Fray, C. Altavilla, and E. Ciliberto, Inorganic Nanoparticles (CRC Press, Boca Raton, FL, 2010), pp. 33-68.

[4] J. Penuelas, A. Ouerghi, C. Andreazza-Vignolle, J. Gierak, E. Bourhis, P. Andreazza, J. Kiermaier, and T. Sauvage, Local tuning of CoPt nanoparticle size and density with a focused ion beam nanowriter, Nanotechnology 20, 425304 (2009).

[5] A. H. Reid, X. Shen, P. Maldonado, T. Chase, E. Jal, P. W. Granitzka, K. Carva, R. K. Li, J. Li, L. Wu, T. Vecchione, T. Liu, Z. Chen, D. J. Higley, N. Hartmann, R. Coffee, J. Wu, G. L. Dakovski, W. F. Schlotter, H. Ohldag, Y. K. Takahashi, V. Mehta, O. Hellwig, A. Fry, Y. Zhu, J. Cao, E. E. Fullerton, J. Stöhr, P. M. Oppeneer, X. J. Wang, and H. A. Dürr, Beyond a phenomenological description of magnetostriction, Nat. Commun. 9, 388 (2018).

[6] G. Wu, K. More, C. Johnston, and P. Zelenay, Highperformance electrocatalysts for oxygen reduction derived from polyaniline, iron, and cobalt, Science 332, 443 (2011).

[7] Y. Liang, Y. Li, H. Wang, J. Zhou, J. Wang, T. Regier, and H. Dai, $\mathrm{Co}_{3} \mathrm{O}_{4}$ nanocrystals on graphene as a synergistic catalyst for oxygen reduction reaction, Nat. Mater. 10, 780 (2011).

[8] D. Wang, H. L. Xin, R. Hovden, H. Wang, Y. Yu, D. A. Muller, F. J. Disalvo, and H. D. Abruña, Structurally ordered intermetallic platinum-cobalt core-shell nanoparticles with enhanced activity and stability as oxygen reduction electrocatalysts, Nat. Mater. 12, 81 (2013).

[9] A. Roucoux, J. Schulz, and H. Patin, Reduced transition metal colloids: A novel family of reusable catalysts? Chem. Rev. 102, 3757 (2002).

[10] S. Guo, S. Zhang, L. Wu, and S. Sun, Co/CoO nanoparticles assembled on graphene for electrochemical reduction of oxygen, Angew. Chem., Int. Ed. 51, 11770 (2012).

[11] C. Petit, Z. L. Wang, and M. P. Pileni, Ferromagnetic cobalt nanocrystals achieved by soft annealing approach-From individual behavior to mesoscopic organized properties, J. Magn. Magn. Mater. 312, 390 (2007).

[12] C. J. Rodrigues, J. A. Bobb, M. G. John, S. P. Fisenko, M. S. El-Shall, and K. M. Tibbetts, Nucleation and growth of gold nanoparticles initiated by nanosecond and femtosecond laser irradiation of aqueous [AuCl4]-, Phys. Chem. Chem. Phys. 20, 28465 (2018).

[13] A. Plech, V. Kotaidis, S. Grésillon, C. Dahmen, and G. von Plessen, Laser-induced heating and melting of gold nanoparticles studied by time-resolved x-ray scattering, Phys. Rev. B 70, 195423 (2004).
[14] A. Plech, V. Kotaidis, K. Istomin, and M. Wulff, Smallangle pump-probe studies of photoexcited nanoparticles, J. Synchrotron Radiat. 14, 288 (2007).

[15] Y. Ihm, D. H. Cho, D. Sung, D. Nam, C. Jung, T. Sato, S. Kim, J. Park, S. Kim, M. Gallagher-Jones, Y. Kim, R. Xu, S. Owada, J. H. Shim, K. Tono, M. Yabashi, T. Ishikawa, J. Miao, D. Y. Noh, and C. Song, Direct observation of picosecond melting and disintegration of metallic nanoparticles, Nat. Commun. 10, 2411 (2019).

[16] D. A. Young, Phase Diagrams of the Elements-UCRL-51902 (U.S. Energy Research and Development Administration, Livermore, CA, 1975).

[17] M. Erbudak, E. Wetli, M. Hochstrasser, D. Pescia, and D. D. Vvedensky, Surface Phase Transitions during Martensitic Transformations of Single-Crystal Co, Phys. Rev. Lett. 79, 1893 (1997).

[18] R. Bauer, E. A. Jägle, W. Baumann, and E. J. Mittemeijer, Kinetics of the allotropic hcp-fcc phase transformation in cobalt, Philos. Mag. 91, 437 (2011).

[19] V. Puntes, K. Krishnan, and A. P. Alivasatos, Colloidal nanocrystal shape and size control: The case of Cobalt, Science 291, 2115 (2001).

[20] D. P. Dinega, Synthesis and characterization of cobalt nanocrystals, Ph.D. thesis, Massachusetts Institute of Technology, 2001.

[21] M. V. Izquierdo, Synthesis of nanoparticles and nanostructured materials by self-assembly, Ph.D. thesis, Universitat Autonoma Barcelona, 2012.

[22] E. Zacharaki, M. Kalyva, H. Fjellvåg, and A. O. Sjåstad, Burst nucleation by hot injection for size controlled synthesis of $\epsilon$ cobalt nanoparticles, Chem. Cent. J. 10, 10 (2016).

[23] S. Sun and C. B. Murray, Synthesis of monodisperse cobalt nanocrystals and their assembly into magnetic superlattices (invited), J. Appl. Phys. 85, 4325 (1999).

[24] G. Schmid, Nanoparticles: From Theory to Applications, 2nd ed. (Wiley-VCH Verlag, Weinheim, 2010).

[25] V. V. Matveev, D. A. Baranov, G. Y. Yurkov, N. G. Akatiev, I. P. Dotsenko, and S. P. Gubin, Cobalt nanoparticles with preferential hcp structure: A confirmation by X-ray diffraction and NMR, Chem. Phys. Lett. 422, 402 (2006).

[26] D. J. Sprouster and M. C. Ridgway, Ion beam formation and modification of cobalt nanoparticles, Appl. Sci. 2, 396 (2012).

[27] O. Kitakami, H. Sato, Y. Shimada, F. Sato, and M. Tanaka, Size effect on the crystal phase of cobalt fine particles, Phys. Rev. B 56, 13849 (1997).

[28] Y. B. Ji and H. S. Park, Structural and morphological changes of Co nanoparticles and Au-10at.\%Pd thin film studied by in situ heating in a transmission electron microscope, Appl. Microsc. 47, 208 (2017).

[29] D. J. Sprouster, R. Giulian, L. L. Araujo, P. Kluth, B. Johannessen, N. Kirby, K. Nordlund, and M. C. Ridgway, 
Ion-irradiation-induced amorphization of cobalt nanoparticles, Phys. Rev. B 81, 155414 (2010).

[30] D. J. Sprouster, R. Giulian, C. S. Schnohr, L. L. Araujo, P. Kluth, A. P. Byrne, G. J. Foran, B. Johannessen, and M. C. Ridgway, fcc-hcp phase transformation in Co nanoparticles induced by swift heavy-ion irradiation, Phys. Rev. B 80, 115438 (2009).

[31] D. Zhang and S. Chaudhuri, Solidification dynamics and microstructure evolution in nanocrystalline cobalt, Comput. Mater. Sci. 160, 222 (2019).

[32] K. Haldrup, M. Christensen, and M. Meedom Nielsen, Analysis of time-resolved X-ray scattering data from solution-state systems, Acta Crystallogr. A 66, 261 (2010).

[33] K. H. Kim, J. Kim, J. H. Lee, and H. Ihee, Topical Review: Molecular reaction and solvation visualized by time-resolved $\mathrm{X}$-ray solution scattering: Structure, dynamics, and their solvent dependence, Struct. Dynam. 1, 011301 (2014).

[34] J. C. Labiche, O. Mathon, S. Pascarelli, M. A. Newton, G. G. Ferre, C. Curfs, G. Vaughan, A. Homs, and D. F. Carreiras, Invited article: The fast readout low noise camera as a versatile $\mathrm{x}$-ray detector for time resolved dispersive extended $\mathrm{x}$-ray absorption fine structure and diffraction studies of dynamic problems in materials science, chemistry, and catalysis, Rev. Sci. Instrum. 78, 091301 (2007).

[35] See Supplemental Material at http://link.aps.org/supplemental/ 10.1103/PhysRevB.100.245425 for a full X-ray scattering signal and details of the scattering simulations.

[36] K. Haldrup, T. Harlang, M. Christensen, A. Dohn, T. B. Van Driel, K. S. Kjær, N. Harrit, J. Vibenholt, L. Guerin, M. Wulff, and M. M. Nielsen, Bond shortening (1.4 $\AA$ ) in the singlet and triplet excited states of $\left[\mathrm{Ir}_{2}(\text { dimen })_{4}\right]^{2+}$ in solution determined by time-resolved X-ray scattering, Inorg. Chem. 50, 9329 (2011).

[37] J. H. Lee, M. Wulff, S. Bratos, J. Petersen, L. Guerin, J. C. Leicknam, M. Cammarata, Q. Kong, J. Kim, K. B. Møller, and $\mathrm{H}$. Ihee, Filming the birth of molecules and accompanying solvent rearrangement, J. Am. Chem. Soc. 135, 3255 (2013).

[38] M. Cammarata, M. Lorenc, T. K. Kim, J. H. Lee, Q. Y. Kong, E. Pontecorvo, M. Lo Russo, G. Schiró, A. Cupane, M. Wulff, and $\mathrm{H}$. Ihee, Impulsive solvent heating probed by picosecond $\mathrm{x}$-ray diffraction, J. Chem. Phys. 124, 124504 (2006).

[39] K. S. Kjær, T. B. Van Driel, J. Kehres, K. Haldrup, D. Khakhulin, K. Bechgaard, M. Cammarata, M. Wulff, T. J. Sørensen, and M. M. Nielsen, Introducing a standard method for experimental determination of the solvent response in laser pump, X-ray probe time-resolved wide-angle X-ray scattering experiments on systems in solution, Phys. Chem. Chem. Phys. 15, 15003 (2013).

[40] D. P. Dinega and M. G. Bawendi, A solution-phase chemical approach to a new crystal structure of cobalt, Angew. Chem. 38, 1788 (1999).

[41] J. Als-Nielsen and D. McMorrow, Elements of Modern X-ray Physics (John Wiley \& Sons, New York, 2011).

[42] V. Kotaidis and A. Plech, Cavitation dynamics on the nanoscale, Appl. Phys. Lett. 87, 213102 (2005).

[43] V. Kotaidis, C. Dahmen, G. Von Plessen, F. Springer, and A. Plech, Excitation of nanoscale vapor bubbles at the surface of gold nanoparticles in water, J. Chem. Phys. 124, 184702 (2006).

[44] A. M. March, Z. Németh, G. Doumy, A. Andersen, A. A. Haddad, M. F. Tu, Y. Kumagai, J. Bang, C. Bostedt, G. Vankó, D. R. Nascimento, T. A. Assefa, W. Gawelda, N. Govind, and L. Young, Elucidation of the photoaquation reaction mechanism in ferrous hexacyanide using synchrotron x-rays with sub-pulseduration sensitivity, J. Chem. Phys. 151, 144306 (2019).

[45] F. Ewald (ESRF Diagnostics Group) (private communication, 2019)

[46] M. S. Kirschner, D. C. Hannah, B. T. Diroll, X. Zhang, M. J. Wagner, D. Hayes, A. Y. Chang, C. E. Rowland, C. M. Lethiec, G. C. Schatz, L. X. Chen, and R. D. Schaller, Transient melting and recrystallization of semiconductor nanocrystals under multiple electron-hole pair excitation, Nano Lett. 17, 5315 (2017).

[47] M. Hu and G. V. Hartland, Heat dissipation for Au particles in aqueous solution: Relaxation time versus size, J. Phys. Chem. B 106, 7029 (2002).

[48] V. Juvé, M. Scardamaglia, P. Maioli, A. Crut, S. Merabia, L. Joly, N. Del Fatti, and F. Vallée, Cooling dynamics and thermal interface resistance of glass-embedded metal nanoparticles, Phys. Rev. B 80, 195406 (2019).

[49] R. Dimper, H. Reichert, P. Raimondi, L. S. Ortiz, F. Sette, and J. Susini, ESRF Upgrade Program Phase II (2015-2022) - Technical Design Study, The Orange Book (ESRF, Grenoble, 2015).

[50] P. Vester, I. A. Zaluzhnyy, R. P. Kurta, K. B. Moeller, E. Biasin, K. Haldrup, M. M. Nielsen, and I. A. Vartanyants, Ultrafast structural dynamics of photo-reactions revealed by modelindependent X-ray cross-correlation analysis, Struct. Dynam. 6, 024301 (2019). 